\title{
Disabling Hip Osteoarthritis and Cardiovascular Pathology: Presence and Impact
}

\author{
Ray Marks \\ Department of Health \& Behavior Studies, Columbia University, Teachers College, New York, USA \\ E-mail:rm226@columbia.edu
}

\begin{abstract}
Objective: This study examined: 1) the extent to which cases with disabling end-stage hip osteoarthritis would exhibit co-occurring cardiovascular health conditions; 2) the pre and post-surgical impact of the presence of one or more of these health conditions on walking ability. Methods: First, a frequency analysis of the various cardiovascular health conditions listed among 1000 end-stage hip osteoarthritis cases was conducted. Second, a sub-sample of 823 uncomplicated surgical cases was specifically examined. Third, a matched sub-sample of 60 cases with and without cooccurring cardiovascular conditions were compared as regards pain, and their ability to functional physically before and after surgery. Results: Almost 50\% of cases initially examined had one or more cardiovascular conditions. Among the 10 categories listed, the most common was high blood pressure, found in $28 \%$ cases. Significant $(\mathrm{p}<.05)$ associations between having one or more cardiovascular comorbidities and high body mass indices were observed, and co-occurring cardiovascular disease independently predicted walking capacity before and after surgery. Key conclusions: Patients with disabling hip osteoarthritis may exhibit high rates of comorbid cardiovascular health conditions. Those presenting with one or more of cardiovascular condition, may have poorer mobility, both before and after surgery than those with no cardiovascular conditions.
\end{abstract}

Keywords: cardiovascular disease, function, hip joint, osteoarthritis, pain.

\section{Introduction}

Hip osteoarthritis, a prevalent medical condition, causes considerable distress and chronic disability among communitydwelling adults aged 55 years and older, worldwide. Associated with substantial activity limitations, direct as well as indirect financial and social costs, a number of factors other than aging may influence its impact. These factors include but are not limited to the presence of obesity, proprioceptive disturbances, diminished muscle strength and hip joint mobility, and varying degrees of pain [2]. In addition, the presence of one or more comorbid health conditions [1], variations in disease presentation [3], gender [4,5], as well as prior injury or systemic factors, may impact the severity of osteoarthritis [5].

Because hip osteoarthritis, a leading cause of functional disability [4] related to poor-self related health [6] is often approached in a very uniform manner as far as conservative or surgical treatment approaches are concerned [7], even though its severity can vary widely [8], it was felt a better understanding of the disease and its clinical heterogeneity [7] would be useful in attempts to prevent excess disability and maximize intervention outcomes for the affected individual. In particular, because myocardial infarction due to ischemic heart disease has been shown to have the highest impact on self-reported health status across studies with a pooled OR of 3.9, followed by arthroses which has a medium impact according to Diedrichs et al. [9] and vascular conditions such as venous ulcers, along with certain aneurysms [10] can co-occur with osteoarthritis [11] it was felt that if these or related cardiovascular conditions, which appear potentially important albeit often overlooked subsets in the expression of the disease could be identified, more targeted efforts to identify and target these factors in future studies might prove helpful. Emerging data also reveal that obesity, a wellestablished risk factor for osteoarthritis, is also a risk factor for heart disease, and it is possible the sedentary lifestyle of those with cardiovascular health problems increases their risk for osteoarthritis via this pathway, or that osteoarthritis increases the tendency towards overweight, which is a cardiovascular risk factor [12, 13]. However, factors such as cardiovascular disease defined as diseases of the heart and blood vessels, which are very common diseases affecting adults over 60 years of age, and the nature of their interaction with disabling osteoarthritic pain and dysfunction and weight presentation where present have received only a very limited degree of attention in the related literature [3]. 
Thus even though osteoarthritis and its pathology may be influenced at any point in its natural history by this common health condition, including changes in the vascular pattern [14], and both health conditions may share common risk factors such as metabolic disturbances, and suboptimal lifestyle practices, few studies have examined to what extent individuals with end stage hip joint osteoarthritis are likely to suffer from one or more cardiovascular health conditions, whether this condition occurs in sizeable numbers, and whether the presence of any of these pre-existing cardiovascular health conditions appears to interact with weight status, and the average pain and degree of disability experienced by this population.

To reduce this knowledge gap, this study examined the degree to which cases diagnosed as having end stage hip osteoarthritis requiring surgery reported having a co-occurring cardiovascular condition, and whether having one or more cardiovascular conditions correlated with overall function, weight status and the pain experience in a dose dependent way. It was hypothesized that due to their high ages, a reasonably high percentage of cases presently studied would exhibit dual diagnoses of hip osteoarthritis and one or cardiovascular conditions. Since comorbidity is a predictor of hip surgery outcomes, it was believed the presence of one or more cardiovascular conditions would impair their function both before and after surgery to a greater extent than having no such conditions. It was specifically felt that this work would be worthwhile in light of prevailing evidence that osteoarthritis appears to have a strong linkage with metabolic syndrome factors such as obesity and hypertension, plus low physical activity levels [12]. In addition, it was felt that if the presence of one or more cardiovascular health conditions increases the likelihood of hip osteoarthritis cases being more impaired physically than those who have no comorbid condition, treating the vascular related risk factors and taking into account the effect of vascularity on healing, inflammation and function in general, might permit more favourable outcomes for a reasonable proportion of hip osteoarthritis cases before and after surgery as outlined by Marcovic-Denic et al. [15]. More specifically, this analysis attempted to quantify the extent to which cardiovascular conditions would be expected to occur among cases with end stage hip osteoarthritis and to discern if there are any clinically relevant differences in the pain experience, weight, and functional status of these individuals who have one or more than one such condition compared to those who have no such documented condition.

The primary research questions driving this exploratory investigation were the following:

1. How prevalent are vascular-related comorbid health conditions when observed among cases with end stage hip osteoarthritis requiring hip replacement related surgery?

2. What are the most common coexisting cardiovascular conditions observed among community dwelling adults with end stage hip joint osteoarthritis requiring hip replacement surgery?

3. How frequently do one or more of these conditions occur among this group?

4. Is the extent of the disability experienced by experienced by adults with disabling hip joint osteoarthritis similar before and immediately after surgery, regardless of vascular status?

5. Do numbers of vascular conditions affect pre and post surgical status in adults with disabling hip osteoarthritis requiring surgery?

\section{Methods}

\subsection{Sample}

In this retrospective analysis, all available records of 1040 patients hospitalized for some form of hip replacement or revision surgery due to clinically and radiographically diagnosed end-stage hip osteoarthritis of one or both hips or hip fracture including those with a history of previous surgeries for hip osteoarthritis were utilized. These data had been collected prospectively over a 10 month period as part of an approved parent study of hip joint surgical outcomes, but with no active patient involvement in an attempt to recruit a disease free healthy eligible sample.

After excluding those with rheumatological and acute hip fracture diagnoses that could confound the study results, there were 1,000 available records of men and women between the ages of 23-89 years with definitive clinical and radiographic evidence of osteoarthritis of one or both hip joints, among whom 823 had no history of prior surgical complications requiring either revision or restorative secondary surgeries. The majority came from the local community and all were awaiting some form of primary or secondary hip joint surgery due to their prevailing hip osteoarthritis disability.

\subsection{Procedures}

Vascular conditions as noted on the patient's medical record were sought for all 1040 patients and recorded as being present if a history of ischemic heart disease, angina pectoris, stroke, heart failure, thrombosis, intermittent claudication, hypertension, cardiac disease, peripheral vascular disease, or lower extremity arteriol disease was listed following Odding et al [16] and VanDijk et al [2]. Those diagnosed as having avascular necrosis were also noted. Other data 
collected included estimates of symptomatic baseline pain perceptions on a 4-point ordinal scale where 0 was no pain, 1 was mild pain, 2 was moderate pain, and 3 was severe pain. They also included physician's estimates of muscle strength capacity of the affected limb as identified by manual muscle tests of the knee and hip muscles on a 0-5 point ordinal scale, where 0 is no strength capacity and 5 is good isometric strength capacity; hip flexion and hip internal rotation joint range of motion estimates in degrees, self-reported stair climbing ability on a 3-item categorical scale (reciprocal, non-reciprocal, unable), assistive device usage (yes or no), plus self-reported maximum walking distance estimates in blocks was recorded. Separate items on the chart included those of age, gender, body mass index (an indicator of body mass derived from the formula weight/height ${ }^{2}$ ), walking distance recorded on Day 1 post-surgery (in meters), and other listed comorbid health conditions. The data were then systematically entered into SPSS version 16.0 files in order to describe the composite sample and selected sub-samples and to analyze trends in the data as regards comorbid cardiovascular status, in general, along with pain and selected functional variables. Additional analyses were undertaken to compare an age and gender matched sub-group with and without vascular comorbidities as regards key study outcomes using chi-square tests, cross-tabulations, and analysis of variance. An a priori significance level of 0.05 was adopted.

\section{Results}

\subsection{Study Population}

The 1040 cases presently examined had a mean age of $65.36+13.04$ years, with a median age of 68 years, and a mode of 71 years. Approximately $30 \%$ or 312 were 60 years or under in age, and there were more females than males (60\% vs $40 \%$ ). For the cohort as a whole, there were 9 distinct comorbid health domains related to different body systems reported as outlined by Dyke et al [1], excluding obesity. The frequency of patients having reported either a mental or physical pre-existing health condition ranged from 0 or no co-existing problem to 5, with a median of $1 ; 36 \%$ had no comorbid condition, $30.5 \%$ had 1 condition, $22.9 \%$ had 2 conditions, $6.7 \%$ had 3 conditions, $1.2 \%$ had 4 health conditions, and .2 had 5 co-existing conditions. Thus a sizeable majority or $69.5 \%$ of cases had at least one additional pre-existing physical health condition and the numbers of reported comorbid health conditions increased with age $(\mathrm{r}=.232 ; \mathrm{p}=.001)$ (See Table 1). Among those with comorbid vascular health conditions, other than avascular necrosis, present in 20 cases, the most commonly observed condition was hypertension alone, or hypertension in combination with coronary artery or cardiac disease.

Table 1: Demographic and related characteristics of cohort ( $\mathrm{N}=1039)$. Values denote numbers, means, standard deviations, and percentages.

\begin{tabular}{|l|l|l|}
\hline Variables & Values & Percentages \\
\hline All patients & & \\
Age (years) & $65.36 \pm 1$ & \\
Under 60 & 316 & 30.4 \\
Over 60 & 723 & 69.6 \\
& & \\
\hline Gender & & \\
Male & 437 & 42.3 \\
Female & 600 & 57.7 \\
\hline Body mass index $\left(\mathrm{kg} \cdot \mathrm{m}^{-2}\right)$ & $27.5 \pm 5.7$ & 37 \\
\hline Morbidity Count & $1.01 \pm 1.0$ & \\
& 384 & 37 \\
1 & 324 & 31.2 \\
2 & 243 & 23.4 \\
3 & 71 & 6.8 \\
4 & 13 & 1.3 \\
\hline
\end{tabular}

After excluding 30 cases with concomitant rheumatoid arthritis, and 10 acute hip fracture cases from the sample of 1040 patients, given that these conditions can cause excess disability due to vascular and other related factors, it was observed that 244 cases or approximately $24 \%$ of the cohort had one or more of the aforementioned vascular conditions. This did not include high blood pressure, which alone was present in 280 cases or $28 \%$ of the subjects, suggesting the prevalence of one or more cardiovascular conditions exceeded 50\% among those with a specific hip osteoarthritis diagnosis (See Table 2). 
Table 2: Frequency distribution of the observed vascular related conditions $(\mathrm{N}=1000)$

\begin{tabular}{lll} 
& Number & Percentage \\
\hline Variables & 244 & 24.4 \\
1 CV Condition & 341 & 34.1 \\
Cypertension & 111 & 11.1 \\
2 or more CV Conditions & 28 & 2.8 \\
\hline
\end{tabular}

$* \mathrm{CV}=$ cardiovascular condition $(\mathrm{s})$ including:

Angina, Aortic aneurism/sclerosis/stenosis/regurgitation, Cardiomegaly, Cardiomyopathy, Congenital Heart Disease, Endocarditis, Stroke, Ischemic heart disease/coronary artery disease/angioplasty**, Myocardial infarction, Heart failure, Heart valve disease/mitral valve prolapse, Heart murmur/palpitations/arrythmias/atrial fibrillation/bradycardia/tachycardia, Left ventricular dysfunction, Pericarditis, Peripheral vascular disease.

** Most commonly reported condition

\subsection{Related Subgroup Analyses}

\subsubsection{Patients with no prior complications}

Among the sub-group of 823 cases derived from the sample of 1000 hip osteoarthritis cases, who were either unilateral primary hip replacement candidates or bilateral hip replacement candidates, whose health status was not compromised by prior complications attributable to hip joint surgery for osteoarthritis (58\% Female;42\% Male), approximately $48 \%$ $(n=390)$ had no comorbid cardiovascular health problem; 52\% $(n=430)$ had 1 cardiovascular problem; 12\% $(n=101)$ had 2 cardiovascular conditions; and $1 \%(n=9)$ had 3 cardiovascular conditions (See Tables 3 and 4 ). Their body mass on average was $26.7 \pm 5.4$ for those with no comorbid conditions, $28.4 \pm 6.1$ for those with one cardiovascular condition; $28.1 \pm 5.4$ for those with two such conditions, and 27.6 \pm 5.7 for those with three conditions of the cardiovascular system, and this was positively correlated with comorbidity numbers $(r=.12, p=.001)$ using a 2-tailed Spearman's rho correlation test. The body mass indices between groups analysis of those with and without a cardiovascular diagnosis revealed this too was significant $(\mathrm{F}=5.197, d f=3, \mathrm{p}=.001)$.

Table 3: Characteristics of cases exhibiting no surgical complications. Values provided are numbers, means, standard deviations, and percentages

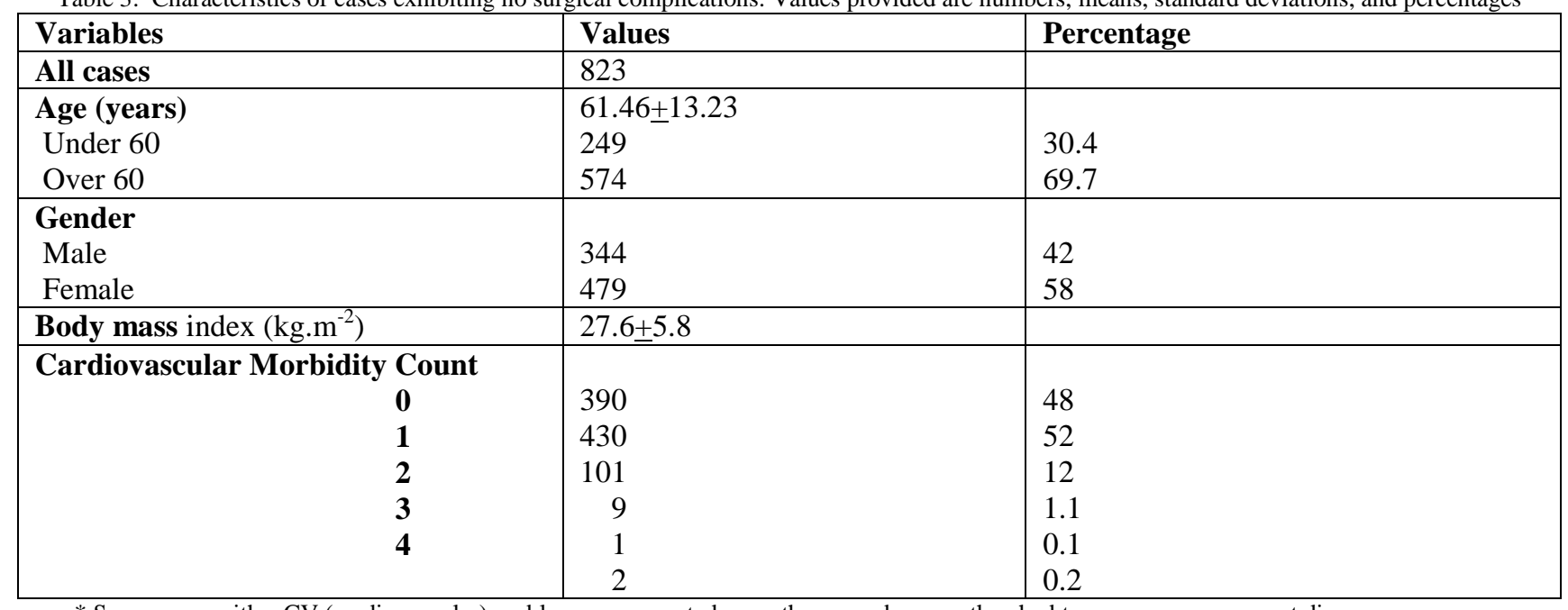

* Some cases with a CV (cardiovascular) problem were counted more than once because they had two or more concurrent diagnoses.

Table 4: Frequency distribution of cardiovascular (CV) related conditions observed plus body mass indices (BMI) characteristics for each diagnostic category $(\mathrm{N}=823)$.

\begin{tabular}{llll}
\hline Diagnostic category & Number & Percentage & Mean BMI(CI) ${ }^{\mathbf{2}}$ \\
\hline *At least 1 CV Condition & 186 & 22 & $26.6(24.8-28.4)$ \\
excluding hypertension & & & \\
Hypertension & & 38 & $29.5(28.2-30.8)$ \\
CV disease + Hypertension & 97 & 11.7 & $27.8(25.7-29.6)$ \\
2 or more CV conditions & 24 & 2.9 & $29(26.5-31.5)$ \\
\hline *Inctudes: & &
\end{tabular}

*Includes: 
Heart murmur/palpitations/arrythmias/atrial fibrillation/bradycardia/tachycardia $\mathrm{N}=57(24 \%)$ cases

Peripheral vascular disease $\mathrm{N}=35(14.7 \%)$ cases

Valve diseases/prolapse $\mathrm{N}=24(10.1 \%)$ cases

Aortic aneurism/sclerosis/stenosis/regurgitation $\mathrm{N}=17(7.1 \%)$ cases

Stroke $\mathrm{N}=6$ cases

Heart failure $\mathrm{N}=3$ cases

Pericarditis $\mathrm{N}=3$ cases

Cardiomegaly/cardiomyopathy $\mathrm{N}=2$ cases

Endocarditis $\mathrm{N}=1$ case

Left ventricular dysfunction $\mathrm{N}=1$ case

1Occurs more frequently than other categories $\mathrm{p}=.001$

2Showing trend towards being higher than body mass indices of those with no health condition BMI 26.1(p=.001)

Note: Overweight=BMI range, 25-29.9

\subsubsection{Matched cases with and without vascular conditions}

In comparing a smaller representative sub-group, mean age 68.2+9.1 years, who presented with one or more vascular condition, with those of a sub-group of comparable ages and gender that did not, walking ability before and immediately after surgery was less efficient in the vascular group, even though cases in the vascular group had only slightly higher percentages of lower leg strength deficits ( $36 \%$ vs $24 \%$ )(Chi-Square $2.286, d f=2, p=.319)$, comparable hip joint range of motion measures, and walking distance at baseline was not associated with age $(\mathrm{r}=-.21 ; \mathrm{p}=.16)$.

In this sub-analysis of a representative age-and gender matched sample of 60 cases, patients with one or more cardiovascular conditions (mean age 68.7 \pm 9.4 years), but no other health conditions, tended to have more pain ( $\mathrm{r}=.27$; $\mathrm{p}=.09)$, and lower presurgical walking ability $(\mathrm{r}=-.35 ; \mathrm{p}=.02)$ than those with no comorbid condition (mean age $67.6 \pm 8.8$ years) (see Table 4 ). For combinations of cardiac and/or vascular diagnoses, excluding avascular necrosis, the subjective pain levels experienced before surgery of $2.50 \pm .63$ vs $2.21 \pm .41(0-4$ scale) were somewhat higher ( $\mathrm{p}=.1)$ for those with a comorbid vascular health problem, even though their disease duration was slightly lower (4.98 vs 5.65 years), and the relationship between the pain scores and number of vascular health conditions was generally unaffected by age $(\mathrm{r}=.27 ; \mathrm{p}=.09)$. As well, the disease free sub-sample tended to be able to walk further on average those with a vascular history on Day 1 post surgery $(2.8 \mathrm{~m}$ vs $5.9 \mathrm{~m})$, who generally could not walk as far and showed less variation in this variable, but this was not significant $(\mathrm{p}=.23)$. While patients had comparable osteoarthritis diagnoses, body mass as defined by the individual's body mass index, was higher in those with a vascular condition, compared to those with no comorbid condition $(28.5 \pm 5.7$ vs $26.7 \pm 4.3)$ and those with higher body mass indices used more assistive devices than those with lower body mass indices $(\mathrm{p}=.001)$. This is of import because although Day 1 post surgical walking distances were statistically comparable, fewer cases with cardiovascular conditions walked as far as those without such conditions and there was an overall significant inverse correlation observed between ideal weight magnitudes, higher in those with cardiovascular comorbidities and walking distance achieved one day after surgery $(\mathrm{r}=-.246, \mathrm{p}=.043)$. Walking endurance at baseline was notably worse if the patients had a pre-existing vascular problem $(\mathrm{p}=.01)$, and distance walked before surgery as reported by the patients was inversely associated with the number of vascular conditions present $(\mathrm{r}=-30 ; \mathrm{p}=.03)$ and device usage $(\mathrm{p}=.01)$, even though device use was comparable among the cohort as a whole. Although the cohort were clearly challenged physically speaking, in that $50 \%$ used assistive devices, and only $23 \%$ could climb stairs reciprocally before surgery, only $20 \%$ of those with vascular conditions could do this compared to $28 \%$ of those with no health condition. Those with vascular disease histories were generally more impaired before and immediately after surgery despite a comparable disease history ( $p=.94)$ (See Tables 5 and 6).

Table 5: Summary of key variables observed among selected age and gender-matched groups from the larger cohort and categorized by presence or absence of vascular disease showing significant differences* based on disease status $\mathrm{p}<0.05$

\begin{tabular}{llll}
\multicolumn{2}{l}{ Varsence of vascular disease showing significant differences* based on disease status p 0.05} \\
& Group I (N=34) & Group II (N=24) \\
& Vascular Condition & No Comorbidity \\
& Present & & P value \\
\hline Age (years) & $68.7 \pm 9.4$ & $67.8 \pm 8.8$ & .58 \\
BMI & $28.5 \pm 5.7$ & $26.7+4.3$ & .34 \\
Gender (\% female) & 60 & 60 & .80 \\
\% using device & 50 & $5.7 \pm 5.9$ & .94 \\
Disease Duration (years) & $5.5 \pm 7.6$ & $3.9 \pm 4.3$ & .27 \\
Internal rotation (degrees) & $5.8 \pm 5.6$ & 0 & $.001 *$ \\
Number of Vascular Diseases & $2 \pm .8$ & $2.2 \pm .4$ & .12 \\
Pain (0-4) & $2.5 \pm .6$ & $5.2 \pm 4.2$ & $.01 *$ \\
Blocks walked before surgery (no.) & $2.5 \pm 2.7$ & $5.9 \pm 13.4$ & .23 \\
Distance walked after surgery (m) & $2.8 \pm 4.5$ & 50 & \\
\hline
\end{tabular}

Note: BMI=body mass index, and a BMI range of 25-29.9 represents the overweight category 
Table 6: Selected partial correlations among study variables showing effect of cardiovascular disease presence (\#) on pre surgical status is independent of age $(\mathrm{N}=60)$

\begin{tabular}{lllllll}
\hline Disease \# & Age & \multicolumn{2}{l}{ Walking Distance Before Surgery } & Walking Distance After Surgery & Pain & \\
\hline $\mathrm{r}$ & .104 & -.35 & $* .30$ & -.62 & .26 & $* 27$ \\
$\mathrm{p}$ & .31 & .02 & .03 & .28 & .09 & .09 \\
\hline
\end{tabular}

*Controlling for age

\section{Discussion}

Hip joint osteoarthritis, a debilitating condition that increases in prevalence with age, presents an enormous challenge to the health care system worldwide due to its chronicity. To improve the outcomes for this group of adults, who may be very hampered as regards activities of daily living involving the lower limb, careful evaluation of all underlying pathophysiological and contributing factors to the disease and its attendant disability has become essential. To this end, this present analysis examined the degree to which adults with hip joint osteoarthritis might exhibit evidence of a comorbid cardiovascular related health condition, an impairment that can adversely influence locomotor ability [16, 20]. It also examined what interrelationships might exist among vascular related correlates and selected disability characteristics of individuals with end stage hip osteoarthritis. In particular, a better understanding of factors such as vascular health status, and the clinical parameters of pain and functional disability was sought, given the immense personal as well as enormous medical costs associated with the presence of osteoarthritis comorbidities [17]. The goal was to examine if adults with hip osteoarthritis would exhibit a high prevalence of co-occurring cardiovascular health conditions, and in light of mounting evidence that vascular pathology can influence osteoarthritic disease progression [27], whether the presence of one or more of these conditions was would be found related to their functional ability. Patients with symptomatic end-stage disabling hip osteoarthritis, requiring surgery were selected as there has been no universal agreement as to what stage of progression is pertinent to examine in the natural history of the disease, and this disease stage has been poorly evaluated in the past [18]. All cases studied thus had significant clinical as well as functional disability that had progressed towards its end stage where it may be helpful to identify specific patient subsets as suggested by Bierma-Zeinstra and Verhagen [7], and whether these have any relationship to correlates found to influence the impairment in general, such as walking ability.

While a retrospective chart review approach can be seen as limitation, and the temporal relationship between the cooccurring conditions could not be teased out, this exploratory study tried to include the entire population of patients with active disease requiring surgery for hip osteoarthritis over a 10 month and to establish the frequency with which these adults had pre-existing cardiovascular health conditions. Although the results of this analysis could be biased by who was studied, in terms of generalizability, the researcher tried to eliminate potential confounders such as autoimmune conditions, and surgical complications. The present age range of the studied cohort was also quite typical of those described in the literature [e.g. 19], and selected analyses were conducted controlling for age. Consistent with several other studies [e.g. 19], 60\% of the present cases were women, a high percentage presented with one or more chronic comorbidities [2], including cardiovascular conditions [10], and the mean body mass index of the cohort was comparable to that of Dijk et al. [2], suggesting this was a reasonably representative sample. In accord with findings of van Dijk et al. [2] and Singh et al. [20], a high percentage of cases had one or more pre-existing cardiovascular health conditions, many had hypertension as described by Nielen et al. [21] and the presence of these conditions impacted pre and postsurgical mobility estimates adversely as identified by Juhakoski et al. [22].

To control for confounding factors, when subjects were carefully matched for age, the finding that Day 1 post surgical functional recovery was more limited in the group with dual diagnoses, implies the presence of one or more cardiovascular conditions can influence the extent of immediate post surgical recovery as assessed by walking distance. Locomotor activity prior to surgery, a predictor of postsurgical status [23] was also more limited in the group with one or more pre-existing cardiovascular conditions and may suggest those who have comorbid cardiovascular disease histories may need more intense post-operative intervention than those who do not. This may be because they are less fit as a whole, or have distinctly altered healing processes, in the presence of arteriol occlusion and/or venous congestion and inflammation [24]. This sub-group was also more overweight, in general, and may have been more unfit as a result at baseline, or subject to heightened degenerative and inflammatory reactions as outlined by Martocchia et al. [25].

Although the cause-effect relationship between osteoarthritis and the comorbidities that can accompany this are unclear [17], it seems plausible to suggest adults with hip joint osteoarthritis who have a co-prevalent cardiovascular condition are likely to suffer to a greater degree from their condition than those who are otherwise healthy. Since their potentially more burdensome disability experience and higher risk of death compared with the general population as a result of their cardiovascular history [29], may be strongly related to their walking ability [29], cognitive, psychosocial or structural factors as a result of one or more of these conditions, clinicians treating these patients may need to monitor this sub group quite closely in order to offset any excessive functional declines attributable to their vascular status. Although medication intake was not controlled for in this analysis, the limited walking ability reported in the presence 
of hip joint osteoarthritis is likely to have an immense negative impact on the life quality of the community dwelling adult if not addressed in the earliest stages of the disease process. Other research similarly emphasizes the importance of early detection and secondary prevention of all cardiovascular problems, to prevent future worsening of activity limitations [2], huge additional medical costs [17] and based on early studies, the risk for developing osteoarthritis [21][See Fig. 1].

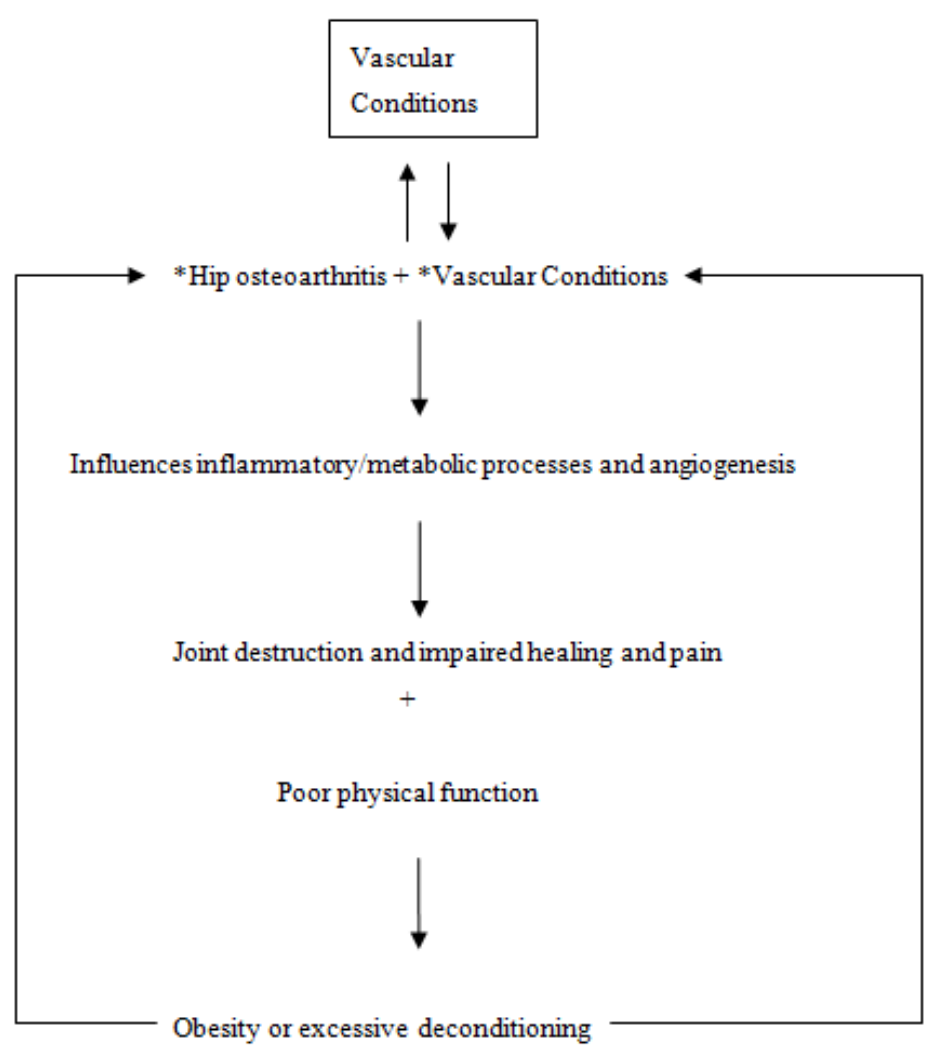

*Common risk and pathogenetic factors for hip osteoarthritis and cardiovascular conditions include:

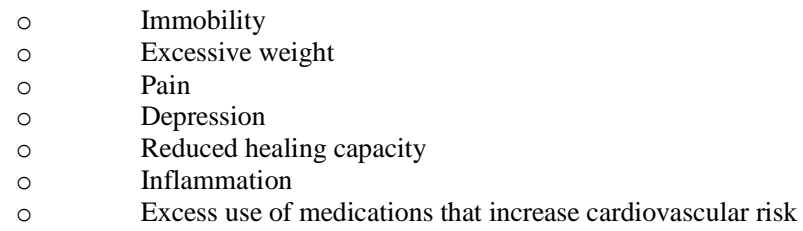

Fig. 1. Hypothetical relationship of risk factors in predicting severe hip osteoarthritis outcomes controlling for age, gender, and body mass index. Adapted from: Findlay [27]; Haywood et al. [28]; Rahman et al. [30]

Moreover, if the level of soluble vascular cell adhesion molecule (VCAM-1) is a possible predictor of severe hip osteoarthritis, and atherosclerosis and thrombosis as well as inflammation [26], sub groups with a high level of this factor might be selectively targeted.

In the interim, it is recognized that the present cohort may not represent all cases with condition; not all data were available or complete; and the use of chart reviews to explore temporal trends and interactions and understand mediating or moderating factors influencing hip osteoarthritis disability may not be without limitations. Also, because only selected types of comorbid vascular problems and their frequency of occurrence were recorded, but not their severity, the impact of these conditions on the disease presentation should be studied further. In the interim, the fact that hip osteoarthritis patients with a vascular condition are likely to be heavier than those who do not should not be ignored, and may explain some of the presently observed variance in functional ability between these two different diagnostic categories, such as device use found to correlate significantly with body mass $(\mathrm{p}=.01)$ and stair climbing ability $(\mathrm{p}=.001)$ should make us more sensitive to the need for better clarity in characterizing the disease.

Furthermore, because the present study showed adults with hip osteoarthritis and one or more cardiovascular conditions tend to have higher rates of leg weakness than healthy patients, the relationship of peripheral vascular disease to ambulatory capacity and life quality of people with hip osteoarthritis may be helpful to explore, especially where adherence issues and safety factors would be important. 


\section{Conclusion}

In accord with other emerging data that vascular pathology may play a role in the pathogenesis of osteoarthritis [26], adults with hip osteoarthritis disability and one or more comorbid vascular health conditions studied here were more disabled than those without vascular pathology. In addition, many of these cases had higher body mass indices on average than those with no comorbid cardiovascular health condition. Since vascular problems, as well as obesity are potential pathogenic factors that influence the natural history of the disease [26, 30-36], professionals interested in reducing the burden of hip joint osteoarthritis might be especially attentive to interventions that can normalize body weights, and reduce the onset or severity of cardiovascular health conditions. Based on the high prevalence of hypertension exhibited by the present cohort, and that this subgroup tended to exhibit higher than average body mass indices, as per Arnoldi et al [14] more studies to examine the overlap between hypertension, intraosseous hypertension, pain, and the primary pathology of osteoarthritis would appear advantageous as well. Finally, further studies to reveal precisely why some cases of hip osteoarthritis appear to be more disabled and/or progress less slowly post surgery than others even though they have the same diagnostic condition may be beneficial, as may longitudinal studies that can follow the disease and its natural history temporally. More intensive primary intervention with more attention to the role of cardiovascular safety of non-steroidal anti-inflammatory drugs, and the impact of cardiovascular drugs on bone health would seem pertinent as well.

\section{References}

[1] G.M. Van Dijk C. Veenhof, G.J. Lankhorst, et al., "Limitations in patients with osteoarthritis of the hip and knee: The relationship with body functions, comorbidity and cognitive function", Disabilityand Rehabilitation, 3, 20, (2009), 1685-1691.

[2] G. M. Van Dijk, C. Veenhof, F. Schellevis, et al., "Comorbidity, limitations in activities and pain in patients with osteoarthritis", BioMedCentral Musculoskeletal Diseases, 9, (2008), 95. doi:10.1186/1471-2474-9-95.

[3] J. Dekker, G.M. Van Dijk, C. Veenhof, "Risk factors for functional decline in osteoarthritis of the hip or knee", Current Opinion in Rheumatology, 21, 5, (2009), 520-524.

[4] M. I. O'Connor, "Osteoarthritis of the hip and knee: sex and gender differences", Orthopedic Clinics of North America, 37, 4, (2006), 559568.

[5] D.T. Felson, "An update on the pathogenesis and epidemiology of osteoarthritis", Radiological Clinics of North America, 42, 1, (2004), 1-9.

[6] F. Cecchi, A. Mannoni, R. S. Molino-Lova et al., "Epidemiology of hip and knee pain in a community based sample of Italian persons aged 65 and older", Osteoarthritis and Cartilage, 16, 9, (2008), 1039-1046.

[7] S. M. A. Bierma-Zeinstra, A. P. Verhagen, "Osteoarthritis subpopulations and implications for clinical trial design", Arthritis Research and Therapy, 13, 2, (2011), 213.

[8] P. Dieppe, A. Judge, S. Williams, et al., "Variations in the pre-operative status of patients coming to primary hip replacement for osteoarthritis in European orthopaedic centres", BioMedCentral Musculoskeletal Disorders, 19, (2009), 19.

[9] C.P. Diederichs, J. Wellmann, D.B. Bartels, et al., "How to weight chronic diseases in multimorbidity indices? Development of a new method on the basis of individual data from five population-based studies", Journal of Clinical Epidemiology, 65, 6, (2012), 679-685.

[10] D. van der Linde D, I.M. van de Laar, A.M. Bertoli-Avella, et al., "Aggressive cardiovascular phenotype of aneurysms-osteoarthritis syndrome caused by pathogenic SMAD3 variants", Journal of the American College of Cardiology, 60, 5, (2012), 397-403.

[11] D. J. Margolis, J. Knauss, W. Bilker, "Medical conditions associated with venous leg ulcers", British Journal of Dermatology, 150, 2, (2004), 267-273.

[12] P.R. Kornaat, R. Sharma, R.J. Van Der Geest, et al., "Positive association between increased popliteal artery vessel wall thickness and generalized osteoarthritis: is OA also part of the metabolic syndrome?", Skeletal Radiology, 38, 12, (2009), 1147-1151.

[13] A. Tukker, T.L. Visscher, H.S. Picavet, "Overweight and health problems of the lower extremities: osteoarthritis, pain and disability", Public Health Nutrition, 12, 3, (2009), 359-368.

[14] C.C. Arnoldi, H. Linderholm, H. Mussbichler, "Venous engorgement and intraosseus hypertension in osteoarthritis of the hip", Journal of Bone and Joint Surgery, 54B, 3, (1972), 409-421.

[15] L. Markovic-Denic, K. Zivkovic, A. Lesic, et al., "Risk factors and distribution of symptomatic venous thromboembolism in total hip and knee replacements: prospective study", International Orthopedics, 36, 6, (2012), 1299-1305.

[16] E. Odding, H.A.Valkenberg, H.J. Stam, et al., "Determinants of locomotor disability in people aged 55 years and over: the Rotterdam Study", European Journal of Epidemiology, 17, 11, (2001), 1033-1041.

[17] M. Gore, K.S. Tai, A. Sadosky, et al., "Clinical comorbidities, treatment patterns, and direct medical costs of patients with osteoarthritis in usual care: a retrospective claims database analysis", Journal of MedicalEconomics, 14,4, (2011), 497-507.

[18] M. Dougados, A. Guegen, M. Mguyen, et al., "Radiological progression of hip osteoarthritis: definition, risk factors and correlations with clinical status", Annals of the Rheumatic Diseases, 55, 6, (1996), 356-362.

[19] J.F. Maillefert, A. Guegen, M. Monreal, et al., "Sex differences in hip osteoarthritis: results of a longitudinal study in 508 patients", Annals of the Rheumatic Diseases, 62, 10, (2003), 931-934.

[20] G. Singh, J.D. Miller, F.H. Lee, et al., "Prevalence of cardiovascular disease risk factors among US adults with self-reported osteoarthritis: data from the third national Health and Nutrition Survey", American Journal of Managed Care, 15, Suppl, (2002), S383-391.

[21] M.M. J. Nielen, A. M. van Sijl M.J.L. Peters, et al., "Cardiovascular disease prevalence in patients with inflammatory arthritis, diabetes mellitus and osteoarthritis: a cross-sectional study in primary care", BioMedCentral Musculoskeletal Disorders, 13, (2012), 150.

[22] R. Juhakoski, S. Tenhonen, T. Anttonen, et al., "Factors affecting self-reported pain and physical function in patients with hip osteoarthritis", Archives of Physical Medicine and Rehabilitation, 89, 6, (2008), 1066-1073.

[23] M. Brandes, M. Ringling, C. Winter, et al., "Changes in physical activity and health-related quality of life during the first year after total knee arthroplasty", Arthritis Care Research, 63, 3, (2011), 328-334. 
[24] H. St. C.C.Addis, R.P. Jepson, J.H. Kellgren, “On the effect of arteriol occlusion and venous congestion upon limb pain”, Clinical Science, 9, (1950), 271-280.

[25] A. Martocchia, L. Toussan, M. Stefanelli, et al., "Association of severity of osteoarthritis and carotid atherosclerosis in patients with metabolic syndrome", Rheumatology, 1 (2011), 1.

[26] G. Schett, S. Kiechi, E. Bonara, et al., "Vascular cell adhesion molecule 1 as a predictor of severe osteoarthritis of the hip and knee joints", Arthritis and Rheumatism, 60, 8, (2009), 2381-2389.

[27] D. M. Findlay, "Vascular pathology and osteoarthritis", Rheumatology, 46, 12, (2007), 1763-1768.

[28] L. Haywood, D. F. McWilliams, C.I. Pearson, et al., "Inflammation and angiogenesis in osteoarthritis", Arthritis and Rheumatism, 48, 8, (2003), 2173-2177.

[29] N. Neusch, P. Dieppe, S. Reichenbach, et al., "All cause and disease specific mortality in patients with knee or hip osteoarthritis: population based cohort study", British Medical Journal, 342, (2011), March 8.

[30] M.M. Rahman, J.A. Kopec, J. Cibere, et al., "The relationship between osteoarthritis and cardiovascular disease in a population health survey: a cross-sectional study", British Medical Journal Open, 3, (2013), 5.

[31] F. C. Breedveld, "Osteoarthritis--the impact of a serious disease", Rheumatology (Oxford), 43, Suppl 1, (2004), S4-8.

[32] T.J. Peters, C. Sanders, P. Dieppe, et al., "Factors associated with change in pain and disability over time: a community-based prospective observational study of hip and knee osteoarthritis", British Journal of General Practice, 55, 512, (2005), 205-211.

[33] E. Yusuf, "Metabolic factors in osteoarthritis: obese people do not walk on their hands", Arthritis Research and Therapy, 14, 4, (2012), 123.

[34] R.A. Puenpatom, T.W. Victor, "Increased prevalence of metabolic syndrome in individuals with osteoarthritis: an analysis of NHANES III data", Postgraduate Medicine, 121, 6, (2009), 9-20.

[35] K. L. Ong, B. J. Wu, B.M. Cheung, et al., "Arthritis: its prevalence, risk factors, and association with cardiovascular diseases in the United States, 1999 to 2008", Annals of Epidemiology, 23, 2, (2013), 80-86.

[36] U. T. Kadam, A. Holmberg, M. Blagojevic, et al., "Risk factors for cardiovascular disease and future osteoarthritis-related arthroplasty: a population-based cohort study in men and women from Malmö, Sweden”, Scandinavian Journal of Rheumatology, 40, 6, (2011), 478-485. 